\title{
Understanding Working Memory for Improving Learning
}

\author{
Nahla Aljojo \\ King Abdulaziz University \\ Faculty of Computing and \\ Information Technology, \\ Information System \\ Department \\ Jeddah, 21382, KSA
}

\author{
Huda Saifuddin \\ King Abdulaziz University \\ Arts and Humanities College, \\ Cognitive Psychology \\ Department \\ Makkah, KSA
}

\author{
Rokaya Abduljalil Abed \\ King Abdulaziz University \\ Faculty of Computing and \\ Information Technology, \\ Information System \\ Department \\ Jeddah, KSA
}

\author{
Abrar Alahmary \\ King Abdulaziz University \\ Faculty of Computing and \\ Information Technology, \\ Information System \\ Department \\ Jeddah, KSA
}

\author{
Amany Aljedaany \\ King Abdulaziz University \\ Faculty of Computing and \\ Information Technology, \\ Information System \\ Department \\ Jeddah, KSA
}

\author{
Rahma Takroni \\ King Abdulaziz University \\ Faculty of Computing and \\ Information Technology, \\ Information System \\ Department \\ Jeddah, KSA
}

\begin{abstract}
A web-based working memory (WM) test system is a management system website that allows students to test their ability and skills in remembering visual patterns. It also enables you to record and store the data for individuals. The system is developed using HTML, PHP and MySQL as a database system to manage and store the data. The system targets several users: children and adults who suffer from attention deficit or learning problems. The main objectives for developing the website are to educate the community on the benefits of performing the working memory test of the activity of the brain and improvements in social skills and improving poor academic and professional performance, especially in maths and reading comprehension. This study implements a set of tasks, testing 59 adults aged 18-24 years of age at King Abdul-Aziz University for testing and measuring WM and cognitive abilities. Results showed tests depended on the age entry by the user. The implications of the test results will help people know their WM level before ascertaining the appropriate suggestions, and to make the test suit our society.
\end{abstract}

Keywords: Web-based Working Memory, Short Term Memory, Baddeley Theory, WM Training, Long Term Memory

\section{INTRODUCTION}

Baddeley and Hitch [1] and Baddeley [2] replaced the concept of the short-term memory (STM) with working memory (WM). The crucial difference was that short-term memory was a simple store for information, while the more modern concept of working memory is assumed to be a cognitive component combining storage, processing and executive control of the cognitive processes at hand.

Baddeley and Hitch defined WM as "a brain system that provides temporary storage and manipulation of the information necessary for complex cognitive tasks" [1].

WM plays an essential role in complex cognition so the strength of working memory is often measured with cognitive tests, such as repeating lists of numbers in reverse order or recalling sequences of dots on a screen. People are constantly throughout life dependent on their memory. The definition of working memory is the ability to keep information on-line, typically for a few seconds .

Memory and learning are inseparable. Therefore, you cannot minimise the importance of memory in learning. Learning allows us to acquire knowledge and gain new skills. The memory is the process through which is achieved storage and retention of this knowledge and these skills: to store and activate what has been learned when we wish to use it again in the future [3].

The connection between WM and learning at least in part why WM capacity appears to predict a wide range of academic abilities, including reading and understanding, and gaining language, maths and thinking skills.

Several studies have found an association between performance on domain-specific WM measures and academic subjects.

Deficits in science and maths are the result of spatial disabilities and weaknesses in acquiring a language and learning English occur due to verbal WM disabilities. so the ability of WM can help the student to limit or contribute to the academic performance by performing some of the training.

Thus, this supports the relationship between WM, learning and academics. Therefore, you can improve the capacity to learn and maintain new information by strengthening the ability of WM. You can then use this improvement for academic achievement. This will improve reading comprehension, and you can use it as a means to improve educational competencies, accounting maths and logic skills [4]. 
Proven by previous studies is that the study of improvements in Visual Attention and Working Memory through a Web-based Cognitive Training Program can improve cognitive abilities after days of training. Thus, Lumos developed Improvement of Visual Attention and Working Memory through a Web-based Cognitive Training Program that makes cognitive training tools accessible to many people and provides benefits for an individual to change and improve their abilities online from home. The program comprises a set of exercises such as Memory Match and Monster Garden to improve attention and proves you can change the capacity of the WM in healthy adults.

"Memory Match is a speeded n-back task where users must compare the current stimulus with those presented previously". In addition, the idea of Monster Garden is to navigate through several stages of the mazes while depending on spatial memory, avoiding obstacles to move to another stage. Also, the number of obstacles increases to provide for more difficult stages. The results of this study indicate that training and improving cognitive abilities such as memory, attention and how well people interact socially, and conducting tasks of daily living from problem-solving to driving, are possible with a web-based application [5][6].

Another study, CogMed Working Memory Training: CogMed WM Training, is based on the many studies designed for individuals with ADHD and/or working memory deficits and focuses in this research about effects on students. The goal of the program is to determine when the WM can be improved so the student can learn multiple skills very easily compared to before. CogMed acts as a start for improved learning, allowing the student to build the cognitive platform needed to learn and achieve success in professional and academic performance [7]. CogMed is the online program content of a group of exercises based on age, so we select/discuss one exercise for every age, for example.

- CogMed JM - for pre-schoolers the game is Animals

- CogMed RM - for school-age the game is visual data link

- CogMed QM - for older adolescents and adults the game is chaos

The results of this study indicate that training can improve WM, which enables the student to solve different problems, especially at poor educational and academic levels and in the case of ADHD [8][9][10]. In addition, the study of Visual Working Memory Capacity for Emotional Facial Expressions to evaluate the ability of visual STM.This study initial stimuli display consisted of six different facial expressions, each of which occupied $3.38^{\circ} \times 2.58^{\circ}$ of visual angle. When generating initial stimuli displays, six pictures were randomly pulled from a set of seven pictures, with the restriction that two or more identical expressions could never be present at the same display. then erased the initial stimuli and presented a blank screen for $500 \mathrm{~ms}$. Then only one image of facial expression is chosen located in one place in the initial display. The results of this study indicate that the memory capacity is estimated through accurate response. It has been found that the capacity of visual memory for emotional facial expressions work equals 3.07, which is high compared with the ability to flip the identities and other visual stimuli [11].

Then there is the study on the Impact of Working Memory Training on Memory Performance in Old-Old Adults. In this study, they investigated whether training WM had a salutary effect on the performance of WM and episodic memory octogenarians. For this study, they developed three variants of WM computerised training missions that seem to be more age-appropriate. All of our WM task variants continuously adjusted to the individual's WM capacity in an adaptive way:
If performing well in a particular task, the task gets harder, but if performance fell below a certain level, the task gets easier. The results of this study appear in WM task variant 1 : substantial improvement was seen: . The results of this study appear in WM task variant 1: substantial improvement was seen: [12].

Finally, the study of measuring developmental changes in WM capacity. Measure the WM performing during scanning the place of circle and identifies to increase capacity of WM that measure brain activity. The test focused on filled circles which appear at the start but when the unfilled circles appear, press the button. It was made to test for visual stimulation and eye movements. The result indicates the difference between the load 3 and load 5 linked with age. WMC correlated with WM capacity and WM activity investigated the interaction between WM load and age. There are visual stimuli in task when load 3 tasks will show the difference in visual simulation [13].

The aim of this study is to develop a web-based WM system to test your ability and skills for remembering visual patterns and training to improve the memory that involves temporary storage by using Baddeley theory (2000) [14] for a working memory test. We are focusing in this study on weakness of WM and its impact on education. Also in this paper we discuss how to measure the rate of WM to keep information for a period of time and cognitive abilities for improving learning. The authors chose 59 students from King Abdul-Aziz University and randomly selected students (females) from the Faculty of Computing and Information Technology from different departments (computer science, information system, information technology) for a test to measure WM and cognitive abilities.

\section{BADDELEY THEORY}

Since Alan Baddeley's WM theory co-developed with Graham Hitch was published in 1974 and based on his previous studies in psychology, he has published on many WM aspects in many journal articles. In addition, he states on the faculty webpage, "My interests are in human memory, neuropsychology and in the practical application of cognitive psychology" [2][14][15].

The term WM is first applicable to computational modelling and animal learning research after changes in cognitive psychology in the book Plans [2][14][15]. In the past, the term WM referred to short term memory (STM) and described the ability to keep information over a brief period of time (seconds) but now the concept of STM has been replaced by WM, which has increased focus on the concept of manipulation of information instead of passive maintenance [16].

The working memory test depends on Baddeley theory whose definition of working memory is the ability to keep information on-line, typically for a few seconds. It can be divided into "primary" memory, with a partial capacity, and "long term" memory. It also depends on different modules such as the Central executive (CE), Phonological loop (PL) and Visuospatial sketchpad, but now we focus in this test on measuring your ability and skills to remember the visual patterns and keep information for a few seconds. For example, some activities that are dependent on memory, such as remembering instructions or solving mathematical problems such as when you want to calculate the sum of numbers or other operations require both processing and storage in the cognitive activities. In addition, reading requires 
understanding of information and retrieval in memory [17]. when individuals suffer from weakness in WM the performing daily activities become difficult, such as can not complete the learning activities and instructions. Thus, it is important to verify whether WM can be improved [18]. However, until recently, this poor WM could not be overcome, but some success in improving performance using WM training confirmed possible change [19].

\section{METHOD}

We met with a psychology expert to learn how to improve WM by providing a collection of tests to assess and develop the fundamental cognitive/mental skills and cognitive skills of a person like WM using visual processing method. We conducted the WM test on students of King Abdul-Aziz University to measure their WM performance.

\subsection{Participants}

The total number of participants was 59 adults aged 18-24 years old. Participants in this research were mostly taken from King Abdul-Aziz University; the experiment was done in (Female section) the Faculty of Computing and Information Technology from different departments $(9$ from information technology, 41 from information system and 9 from computer science department) for testing and measuring WM and cognitive abilities.

\subsection{Instruments}

The task of one of the types of WM test that depended on the Baddeley theory was created and run on computers in Lab 118. The test began at 12:00 pm and lasted until 2:00 pm; the number of participants was 59 students from University of king Abdul Aziz, Faculty of Computing and Information Technology in differently departments ( computer science, information system, Information Technology), each group performed the test then the same way for the next group . Before the test, I began speaking about instructional information concerning the aims of the test and the time required to finish it. When they began the test that appeared to them, many pictures were displayed sequentially for 3 seconds. Each time a new picture appeared the more difficulty (increased number of options and shapes) so as to make them more focused on thing, place, shape, meaning they could answer more questions and arrive at a time of higher stage with repetition to develop memory from the STM to WM.

\subsection{Procedure}

The section below expounds how can be using website for procedure Working memory tests and determine and measure of WM by obtain the percentage of abilities.

When they had solved all 40 questions, the results were shown to them.

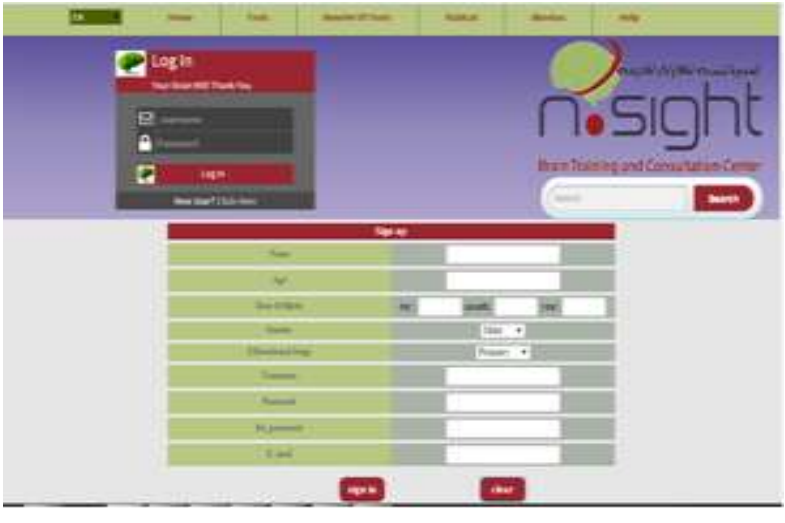

Figure 1: sign up page

\section{- $\quad$ Starting the test:}

The test start page is shown in Figure 2 and this page contains a sign-in form and the button that directs the user to the signin form, and on the left-hand side when clicked then some instructions that contain some of examples of the test appear. Also, the taker had to select the type of payment such as Visa or licence number for admin validation purposes, as shown in Figure 3.

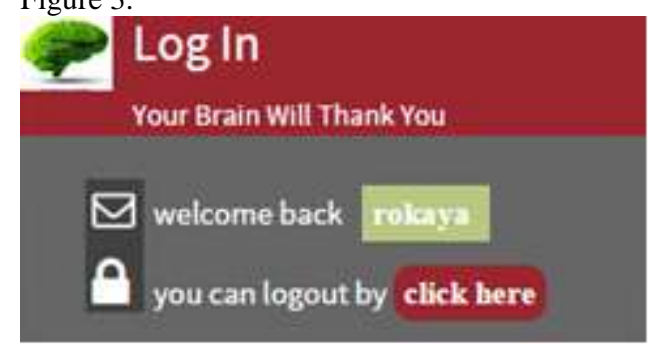

Figure 2. Test start page

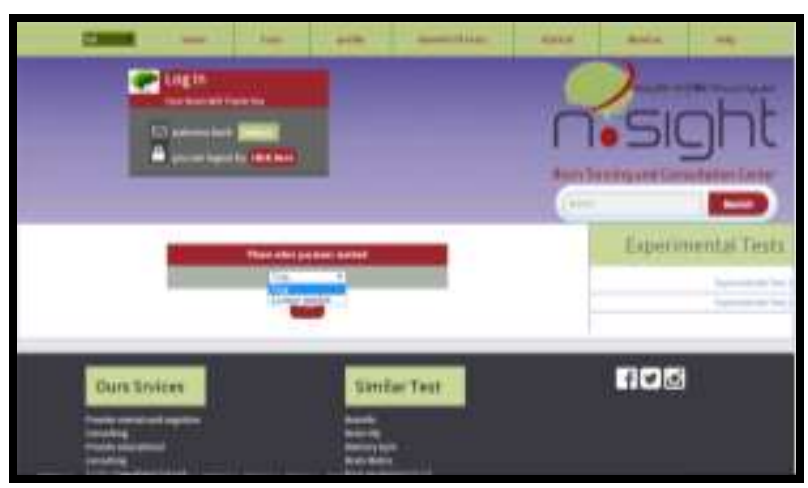

Figure 3. Test start page

Captions should be Times New Roman 9-point bold. They should be numbered (e.g., "Table 1" or "Figure 2"), please note that the word for Table and Figure are spelled out. Figure's captions should be centered beneath the image or 
picture, and Table captions should be centered above the table

\section{Sign-up:}

On this page, the user was required to fill in the registration form and create an account before he/she started the test, as shown in Figure 1.

\section{Test page:}

Now after the taker number is entered at the bottom, click next and click on start the test (see Figures 4,5 ). The test page contains the test and displays a set of questions and every question were displayed a set of pictures sequentially for 3 seconds. Each time a new picture appeared the more difficulty (increased number of options and shapes) so as to make them more focused on thing, place, shape, meaning they could answer more questions and arrive at a time of higher stage with repetition to develop memory from the STM to WM (see Figures 5-13). After answering all 40 questions, the system then calculated the correct answers and displayed two results: the number of correct answers and the percentage depending on the age entry for the user. Also, advice was displayed about the result of the test concerning WM (see Figure 14).

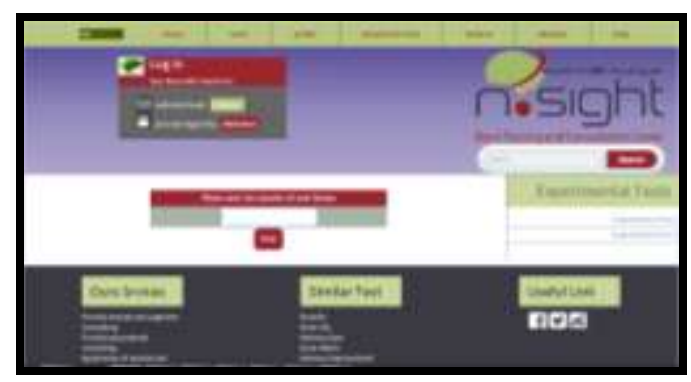

Figure 4: Enter licence number page

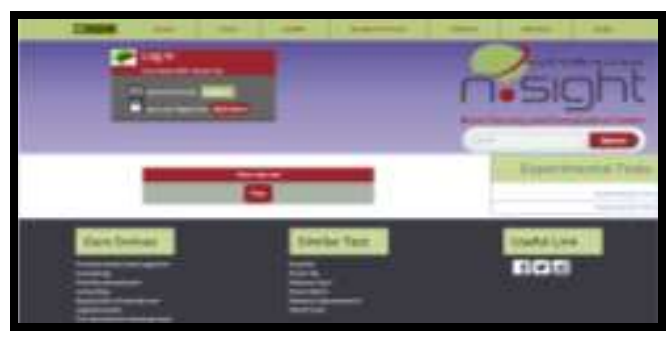

Figure 5: Start the test page

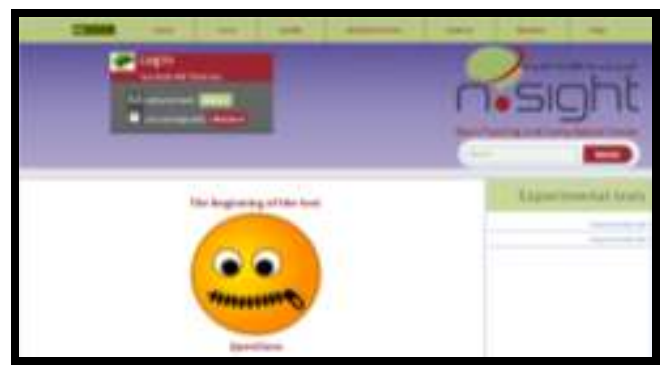

body.

Figure 6: The beginning of the test

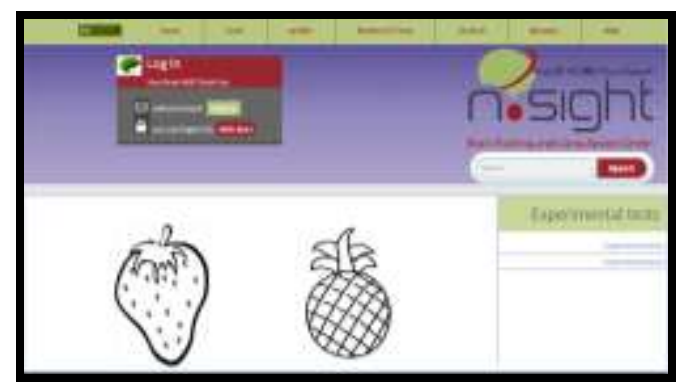

Figure 7: Two images of fruit appear

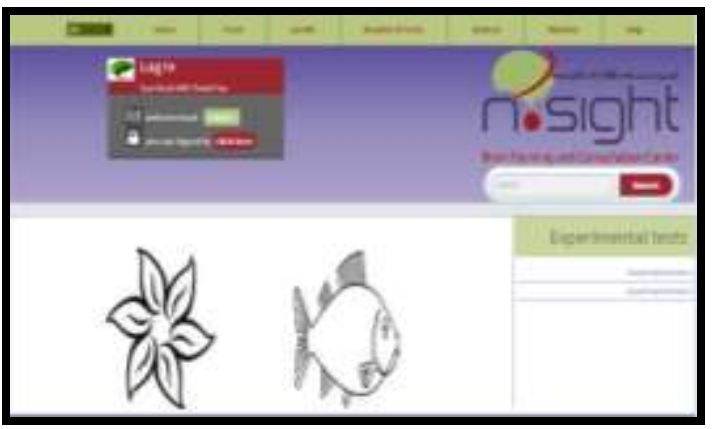

Figure 8: A flower and a fish are displayed

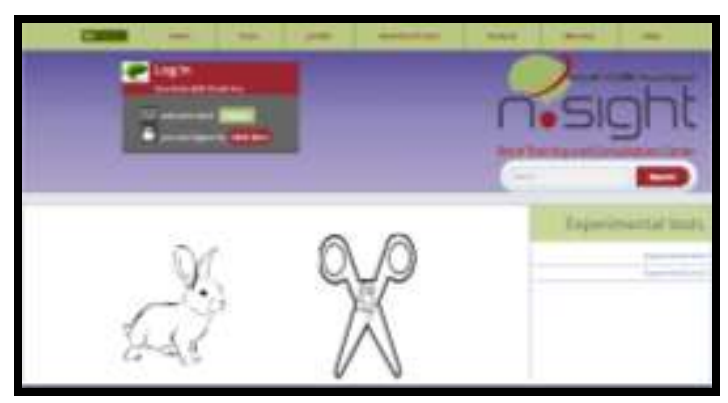

Figure 9: The final two images are shown

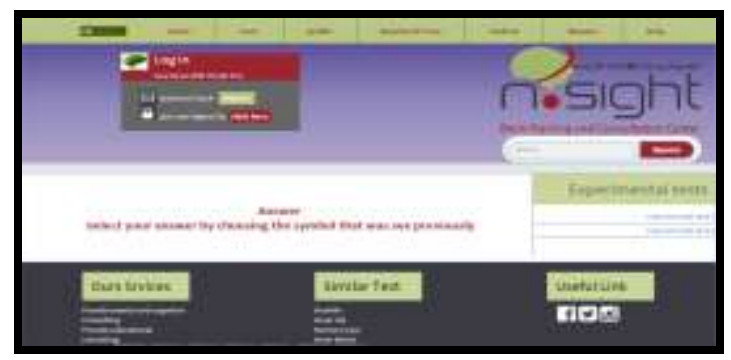

Figure 10: Answer questions 


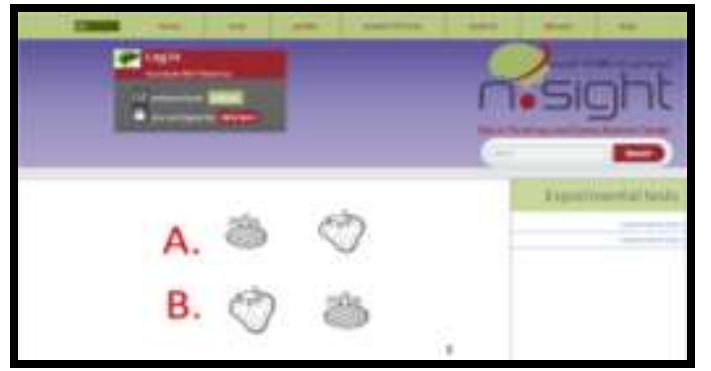

Figure 11: Click answer for picture 1

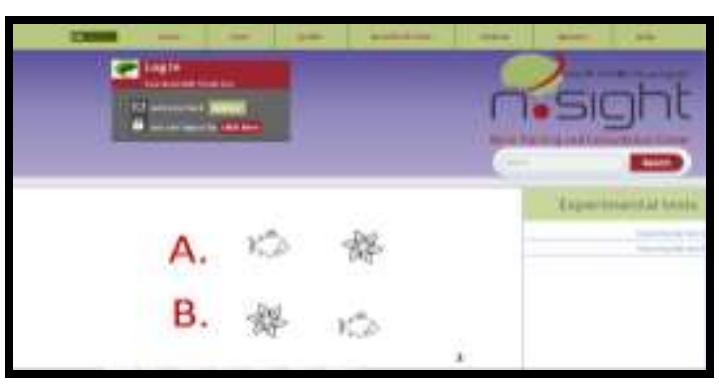

Figure 12: Click answer for picture 2

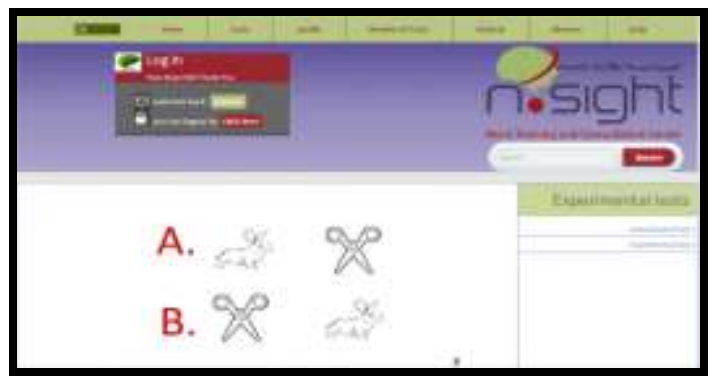

Figure 13: Click answer for picture 3

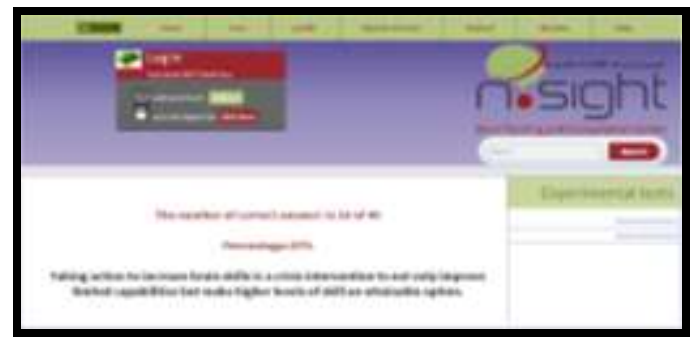

Figure 14: the results of the WM test after answering all questions

\section{RESULTS}

Based on an analysis of the answers, the authors found that Twenty three participants who had the low score (the number of correct answers being less than to the mean). This excepts thirty-six participants who had the high score (the number of correct answers being greater than or equal to the mean). The mean of correct answers for all participants was $=26.3, \mathrm{SD}=$ 4.8 (see Table 1). The correct answers of participants divided into three percentiles were aggregated as follows: see Table 2 .
1) $<=25 \%$ (11 participants that had the correct answers within the $[<=25 \%]$ percentile interval.

2) $>25 \%-50 \%$ (19 participants that had the correct answers within the $[>25 \%-50 \%]$ percentile interval.

3) $>50 \%-75 \%$ (20 participants that had the correct answers within the [ $>50 \%-75 \%]$ percentile interval.

4) $>75 \%$ (9 participants that had the correct answers within the [>75\%] percentile interval.

\section{Table 1: Descriptive statistics}

No_Correct

\begin{tabular}{|ll|r|}
\hline $\mathrm{N}$ & $\begin{array}{l}\text { Valid } \\
\text { Missing }\end{array}$ & 59 \\
Mean & & 0 \\
Median & & 26.3559 \\
Std. Deviation & & 26.0000 \\
Variance & & 4.80934 \\
Percentiles & 25 & 23.130 \\
& 50 & 23.0000 \\
& 75 & 26.0000 \\
& & 30.0000 \\
\hline
\end{tabular}

\begin{tabular}{|c|c|c|c|c|c|}
\hline & & Frequency & Percent & Valid Percent & $\begin{array}{c}\text { Cumulative } \\
\text { Percent }\end{array}$ \\
\hline \multirow[t]{21}{*}{$\begin{array}{l}\text { Valid } \\
\end{array}$} & 18.00 & 1 & 1.7 & 1.7 & 1.7 \\
\hline & 17.00 & 1 & 1.7 & 1.7 & 3.4 \\
\hline & 18.00 & 3 & 5.1 & 5.1 & 8.5 \\
\hline & 19.00 & 1 & 1.7 & 1.7 & 10.2 \\
\hline & 20.00 & 2 & 3.4 & 3.4 & 13.8 \\
\hline & 21.00 & 1 & 1.7 & 1.7 & 15.3 \\
\hline & 22.00 & 2 & 3.4 & 3.4 & 18.6 \\
\hline & 23.00 & 5 & 8.5 & 8.5 & 27.1 \\
\hline & 24.00 & 4 & 6.8 & 6.8 & 33.9 \\
\hline & 25.00 & 3 & 5.1 & 5.1 & 39.0 \\
\hline & 26.00 & 7 & 11.9 & 11.9 & 50.8 \\
\hline & 27.00 & 3 & 5.1 & 5.1 & 55.9 \\
\hline & 28.00 & 5 & 8.5 & 8.5 & 64.4 \\
\hline & 29.00 & 6 & 10.2 & 10.2 & 74.6 \\
\hline & 30.00 & 6 & 10.2 & 10.2 & 84.7 \\
\hline & 31.00 & 4 & 6.8 & 6.8 & 91.5 \\
\hline & 32.00 & 1 & 1.7 & 1.7 & 93.2 \\
\hline & 35.00 & 1 & 1.7 & 1.7 & 94.9 \\
\hline & 36.00 & 2 & 3.4 & 3.4 & 98.3 \\
\hline & 38.00 & 1 & 1.7 & 1.7 & 100.0 \\
\hline & Total & 59 & 100.0 & 100.0 & \\
\hline
\end{tabular}

\section{DISCUSSION}

In discussing the results cited the following themes were evident:

1) $<=25 \%$ (11 participants that had the correct answers within the $[<=25 \%]$ percentile interval. Advice for students: Taking action to increase brain skills is a crisis intervention to not only improve limited capabilities but make higher levels of skill an attainable option.

2) $>25 \%-50 \%$ (19 participants that had the correct answers within the [>25\%-50\% ] percentile interval. Advice for students: Taking action to increase brain skills is a critical 
need to overcome definite weakness, alter mental abilities and allow their capabilities to grow.

3) $>50 \%-75 \%$ (20 participants that had the correct answers within the [>50\%-75\%] percentile interval. Advice for students: Taking action to increase brain skills is a priority based upon personal goals.

4) $>75 \%$ (9 participants that had the correct answers within the [>75\%] percentile. Advice for students: Taking action to increase brain skills is a critical need to overcome definite weakness, alter mental abilities and allow their capabilities to grow.

\section{CONCLUSION}

This paper discussed that impairments of working memory are closely associated with learning deficits, as well as daily classroom activities. Without early intervention, memory deficits cannot be made up over time and will continue to compromise individual's likelihood of academic success. The results may be useful in evaluating their problems, as well as helping them to reduce memory-related failures that lie at the root of substantial learning difficulties, and thus improving the WM is strongly recommended.

\section{FUTURE WORK}

There are many areas that can make the system more efficient by adding greater functionality. For example:

- Activation of payment procedures online by providing payment services such as PayPal, credit card and Visa, etc.

- We used this test on elementary and Bachelor's level students. We hope to apply this test to all levels of education, and to male subjects, too.

- Implementing a smart phone app.

- The system could be applied at a Saudi university for improve learning.

\section{ACKNOWLEDGMENTS}

We would like to thank N.Sight Brain Training and Consultation Centre for supporting and helping in the research.

\section{REFERENCES}

[1] Baddeley, A. D., \& Hitch, G. (1974). Working memory. In G. A. Bower (Ed.), The psychology of learning and motivation:Advances in research and theory (Vol. 8, pp. 47-89). New York: Academic Press.

[2] Baddeley, A. D. (1996). Exploring the central executive. Quarterly Journal of Experimental Psychology, 49A, 528.

[3] Gathercole, S. E., \& Alloway, T. P. (2004). Working memory and classroom learning. Dyslexia Review, 15, 4-9. Grace C. Ashton, Ph.D. (n.d.). Working Memory in the Classroom and Beyond.

[4] Gropper, R. (2013). Working Memory Training In College Students With Attentiondeficit. Ontario Institute For Studies In Education, 129.
[5] Willis, S. L., Tennstedt, S. L., Marsiske, M., Ball, K., Elias, J., Koepke, K. M., Morris, J. N., Rebok, G. W.,...Wright, E. (2006). Long-term effects of cognitive training on everday functional outcomes in older adults. The Journal of the American Medical Association, 296(23), 2805-2814.

[6] Labs, L. (2007). Improvement of Visual Attention and Working Memory through a Web-based Cognitive Training Program.

[7] Westerberg, H., Hirvikoski, T., Forssberg, H., Klingberg, T. (2004). Visuo-spatial working memory: a sensitive measurement of cognitive deficits in ADHD. Child Neuropsychology, 10,155-161.

[8] Westerberg, H., \& Klingberg, T. (2007). Changes in cortical activity after training of working memory - a single-subject analysis. Physiology and Behavior, 92(12), 186- 192. Doi: 10.1016/j.physbeh. 2007.05.041

[9] Lundqvist, A., Gundström, K., \& Rönnberg, J.(2010). Computerized working memory training in a group of patients suffering from acquired brain injury. Brain Injury, 24(10), 1173- 1183.

[10] Johansson, B., \& Tornmalm, M. (2012). Working memory training for patients with acquired brain injury: Effects in daily life. Scandinavian Journal of Occupational Therapy, 19(2), 176-183. doi:10.3109/11038128.2011.603352 .

[11] Domagoj Švegar (2011). Visual Working Memory Capacity for. Emotional Facial Expressions, Psychological Topics 20 (2011), 3, 489-502.

[12] Buschkuehl.M, Jaeggi.S.M, Hutchison.S, PerrigChiello.P, Matthias.C, Mu“ller.M, Breil.F, oppeler.H, Perrig.W (2008). Impact of Working Memory Training on Memory Performance. Psychology and Aging 2008, Vol. 23, No. 4, 743-753.

[13] Klingberg.T, Forssberg.H, Westerberg.H. (2002). Increased Brain Activity in Frontal and Parietal Cortex Underlies the Development of Visuospatial Working Memory Capacity during Childhood. Cognitive Neuroscience, 10.

[14] Baddeley, A. D. (2000). The episodic buVer: a new component of working memory? Trends in Cognitive Sciences, 4, 417-423.

[15] Baddeley, A. D., Gathercole, S. E., \& Papagno, C. (1998). The phonological loop as a language learning device.Psychological Review, 105, 158-173.

[16] Gathercole, S. a. (2008). Working Memory and Learning: A Practical Guide for Teachers. SAGE Publications.

[17] Alloway.T.P. (2006). How does working memory work in the classroom? Educational Research and Reviews Vol. 1 (4), pp. 134-139, July 2006. Available online at http:// www.academicjournals.org/ERR

[18] Alloway, T. P., Gathercole, S. E., \& Pickering, S. J. (2006).Verbal and visuo-spatial short-term and working memory in children: Are they separable? Child Development,77, 1698-1716.

[19] Holmes.J, Gathercole ,S., Dunning. D. (2009). Adaptive training leads to sustained enhancement of poor working memory in children. Blackwell Publishing Ltd, 9600 Garsington Road, Oxford OX4 2DQ, UK and. 